\title{
A Chestnut-A Difficult Challenge for an Endoscopist
}

\author{
Dalia Zykutè ${ }^{1}$ Romanas Zykus² \\ ${ }^{1}$ Department of Gastroenterology, Lithuanian University of Health \\ Sciences, Kaunas, Lithuania \\ ${ }^{2}$ Department of Gastroenterology, Hospital of Lithuanian University \\ of Health Sciences Kaunas Clinics, Kaunas, Lithuania
}

\begin{abstract}
Address for correspondence Dalia Zykutè, MD PhD, Department of Gastroenterology, Lithuanian University of Health Sciences, Mickeviciaus str. 9, Kaunas LT-44307, Lithuania (e-mail: d.zykute@gmail.com).
\end{abstract}

J Digest Endosc 2020;10:240-241
Abstract
Keywords
- unusual foreign body
- esophagus
- chestnut

True foreign bodies are a frequent problem in mentally disabled adults and since ingestion is usually intentional, retrieval can be difficult due to unusual, previously in practice not encountered objects. This case illustrates the laborious effort of removing an esophageal foreign body (FB), a chestnut, in a mentally disabled adult.

\section{Introduction}

Removal of esophageal foreign bodies (FBs) is common practice for endoscopists. In the adult population, the majority of FB ingestions occur while eating; however, any kind of object could be encountered as a FB in the digestive tract. Intentional ingestion of true $\mathrm{FB}$ is especially common among patients with psychiatric disorders; in such cases, the most frequently encountered FB are household items, for example, plastic spoons, toothbrushes, or pens. ${ }^{1-3}$ Occasionally, the management of unusual $\mathrm{FB}$ can be challenging due to little or no coverage in literature. Thus, we report a case of a chestnut in esophagus which required multiple endoscopies to be removed.

\section{Case History}

A 27-year-old male with Down's syndrome, severe mental disability, and past history of two laparotomies for FB removal was transferred due to a chestnut in the lower esophagus after an unsuccessful initial attempt at removing it endoscopically at a regional hospital. Due to the mental condition, the patient could not express any complaints. It was noticed by parents that patient could not swallow food and was choking when trying to ingest water. Patient was afebrile and his physical examination was normal.

Four endoscopies in four days have been performed trying different methods to remove $\mathrm{FB}$, twice under intravenous and twice under general anesthesia. Initial endoscopy revealed wide esophagus and spasm of lower sphincter which raised suspicion that the patient might have achalasia. A $3 \mathrm{~cm}$ in diameter chestnut was located above the lower esophageal sphincter that was not impacted. At first, it was attempted to push the chestnut to the stomach; however, due to sphincter spasm and wide lumen of esophagus, the chestnut would roll with endoscope sliding down the side. Retrieval at this point was futile as well: when trying to grasp the body with a snare, Dormia or lithotripter Dormia basket, it would spin, making grasping impossible, or slip off before it could be clamped firmly. For FB forceps, the chestnut was too large and firm, and a polyp retrieval basket proved to be too shallow and would accommodate only half of the chestnut; thus, a snare would slip off when trying to close the net. Using Foley catheter, it was possible to bring the chestnut up to the upper esophageal sphincter where it was visualized, but the retrieval in this way was not possible because the trajectory of the balloon's force did not match the curve of the esophagus. Therefore, ENT surgeons attempted the removal with rigid instruments; however, while the texture of chestnut is hard and smooth, it breaks off easily. Thus, it was only possible to sever little pieces; consequently, leaving the chestnut with damaged surface. It was decided against removal in this manner, since it would prolong patients' time under general anesthesia greatly. At this point, surgical considerations have been made; however, it was decided to make one more endoscopic attempt. This time we were able to remove the chestnut with a lithotripter Dormia basket, since the damaged surface provided a gripping point and it did not slip off of the instrument (see - Supplementary Figs. S1 and S2 [online only]).
License terms

(두) (1) $\Theta \circledast$ 


\section{Discussion}

Flexible endoscopy is the first line management for $\mathrm{FB}$ retrieval. ${ }^{3-5}$ The failure rate at first attempt of removal has been reported to be 4 to $5.9 \%$, with some of the patients (2.9\%) requiring second endoscopy by a more experienced endoscopist. ${ }^{6,7}$ Surgical approach is required in minority of cases (0.4-3\%), usually due to complications or irretrievable FB. ${ }^{3,6,8}$ It has been reported that in intentional ingestion, surgery rates were higher than accidental ingestions or food impactions. $^{2}$

Grasping forceps, polypectomy snare, basket or retrieval net are all appropriate for successfully securing and retrieving big, blunt, and difficult to grasp FBs., ${ }^{4,5}$ Potentially, the chestnut could have been retrieved with a basket deep enough to accommodate the whole chestnut. Unfortunately, at the time of this particular case, we only had a polyp retrieval basket in our possession; therefore, we did not have a chance to use other retrieval items such as a Roth net. In our case, the problem encountered was inability to grasp due to slipperiness and consistency of the chestnut. Foley catheter is another option that can be used for blunt object (usually coins) removal from esophagus. ${ }^{9}$ Although in our case this method was futile, balloon catheter could be applied as an assisting tool to raise FB and then use other, perhaps more convenient, instruments for removal. In one case report, the chestnut impacted in the upper third of esophagus was removed with a spiral-tipped instrument for myoma fixation, using the corkscrew method..$^{10}$ However, in our case, the chestnut was mobile and above the lower esophageal sphincter; therefore, attempts to utilize the above-described manner could have been hazardous. Even though it is generally recommended to retrieve objects that are bigger than 2 to $2.5 \mathrm{~cm}$, pushing object to the stomach could be another option to have more room for manipulation or leave it to pass spontaneously. ${ }^{5}$ Damaging surface of slippery and difficult-to-retrieve objects at first could be another strategy, since it could provide gripping point for an appropriate instrument; in our case, a mechanical lithotripter Dormia basket.

\section{Conclusion}

The chestnut in our experience is one of the most difficult FBs to manage; nevertheless, with divergent thinking and creativity, it is possible. We presented this rare occurring FB and experience of another case, so that if endoscopists face similar challenge again, the choice of management could be made with more ease.

\section{Conflict of Interest}

None.

\section{References}

1 Conway WC, Sugawa C, Ono H, Lucas CE. Upper GI foreign body: an adult urban emergency hospital experience. Surg Endosc 2007;21(3):455-460

2 Palta R, Sahota A, Bemarki A, Salama P, Simpson N, Laine L. Foreign-body ingestion: characteristics and outcomes in a lower socioeconomic population with predominantly intentional ingestion. Gastrointest Endosc 2009;69(3 Pt 1):426-433

3 Webb WA. Management of foreign bodies of the upper gastrointestinal tract: update. Gastrointest Endosc 1995;41(1):39-51

4 Ikenberry SO, Jue TL, Anderson MA, et al; ASGE Standards of Practice Committee. Management of ingested foreign bodies and food impactions. Gastrointest Endosc 2011;73(6):1085-1091

5 Birk M, Bauerfeind P, Deprez PH, et al. Removal of foreign bodies in the upper gastrointestinal tract in adults: European Society of Gastrointestinal Endoscopy (ESGE) Clinical Guideline. Endoscopy 2016;48(5):489-496

6 Mosca S, Manes G, Martino R, et al. Endoscopic management of foreign bodies in the upper gastrointestinal tract: report on a series of 414 adult patients. Endoscopy 2001;33(8):692-696

7 Li ZS, Sun ZX, Zou DW, Xu GM, Wu RP, Liao Z. Endoscopic management of foreign bodies in the upper-GI tract: experience with 1088 cases in China. Gastrointest Endosc 2006;64(4):485-492

8 Athanassiadi K, Gerazounis M, Metaxas E, Kalantzi N. Management of esophageal foreign bodies: a retrospective review of 400 cases. Eur J Cardiothorac Surg 2002;21(4):653-656

9 Ginsberg GG. Management of ingested foreign objects and food bolus impactions. Gastrointest Endosc 1995;41(1):33-38

10 Matern U, Aschendorff A, Krebs A, Kohlberger E, Rückauer KD. A new method for extracting wooden foreign bodies from the upper esophagus. Endoscopy 2000;32(12):1002-1003 